\title{
Fragmentos de uma história: assim tudo começou... e ainda continua
}

\section{Fragments of a story: so it all began ... And still continues}

Mariantonia Chippari*

\section{Resumo}

A autora relata a história oficial do curso de Psicologia na Universidade Metodista de São Paulo. Baseia-se em documentos internos da instituição e traça sua trajetória, destacando alguns personagens e eventos significativos que ajudaram nessa construção que começou há 45 anos e ainda encontra-se inacabada.

Palavras-chave: curso de Psicologia; Universidade Metodista

\section{Abstract}

The author tells the official history of Psychology course at Methodist University of São Paulo, Brazil. The article is based on institution internal documents and traces this trajectory, highlighting some key persons and events that cooperated for the construction that have begun 45 years ago and is still unfinished.

Keywords: Psychology course; Methodist University

* Psicóloga, mestre em Psicologia pela Pontifícia Universidade Católica de São Paulo. Professora de Métodos de Pesquisa e Psicologia Comportamental. Foi Chefe de Departamento entre os anos de 1996 a 2000 e atualmente é Coordenadora do Curso de Graduação em Psicologia. 
O curso de Psicologia do Instituto Metodista de Ensino Superior iniciou suas atividades em 01/07/1971 e foi autorizado pelo decreto no 68.793 de 22/06/71. Teve seu reconhecimento por meio do decreto lei - 74.259 de $08 / 07 / 74$.

Naquela ocasião, o Diretor Geral do Instituto era o professor Benedito de Paula Bittencourt e o curso de Psicologia estava alocado na Faculdade de Ciências Humanas e Letras. A faculdade tinha como diretor o professor Ely Ezer Cesar.

Em 1975, Prof. Péricles de Oliveira Prado Filho passou a ocupar o cargo de Coordenador do Curso, pois era psicólogo e já estava filiado à instituição, auxiliando na elaboração da matriz curricular e distribuição de disciplinas, além de organizar o funcionamento do curso. Para auxiliar na organização do funcionamento do curso, a coordenação contava com o trabalho de chefes de departamento, escolhidos pelos docentes.

Conforme documentos oficiais (atas e outros relatórios arquivados na instituição, somados às informações fornecidas pelo professor Péricles de Oliveira Prado Filho ${ }^{1}$ ), 53 professores compunham o corpo docente que deu início ao curso. Vários deles também constam da história da Psicologia no Brasil. Listamos a seguir seus nomes:

Agostinho Minicucci; Alvino Augusto de Sá; Anita Liberalesso Neri; Ana Maria Vieira Pires; Antonio Carlos Gil; Antonio Carlos da Silva; Belisário Marques de Andrade; Berta Zylberstajn; Célia Maria Terra Noronha; Cleópatra Poli; Clori Trindade; Dílson Vilela; Doralice Marques; Dorival Rodrigues Beulke; Eda Marconi Custódio; Ely Eser; Eduardo Navajas Filho; Geraldino Pereira Neto; Genésio de Moura Muzel; Gerson Veiga; Gloria Eliza Von Buettner; Geraldo Romanelli; Iara Cândia Chalela; Jaci Marasquim; Jane Persinotti; Joel Gosling; JohannesPetrus Maria Berkers; José João Cury; Ladislau Ruy UngarGlausiusz; Laise de Castro Lira; Luiz Carlos Ferreira; Maria Cecília Grasseschi; Maria Olívia Guedes de Assis; Maria Emília Minicucci; Maria Cecília Veluch Baptista; Maria Aparecida Franco; Maria Luiza Urban de Castro; Maria Salete Dutra da Silva Tomioka;

Agradecimentos ao Prof. Péricles de Oliveira Prado Filho pelas informações que completaram este texto, pois nem todos os detalhes constavam nos documentos oficiais. 
Maria Tereza Nappi; Maria Helena de Barros Berkers; Marilda F. Danna; Marilene Marins de Carvalho; Nilza Musa Licio; Nilo Belotto; Olímpia Rosa Noronha; Osmar Roberto Crivelaro; Paulo Damião; Péricles de Oliveira Prado Filho; Regina M. Moura; SigmarMalvezzi; Suzana Torres; Tereza Bordini; TessaDrexlerRipper.

A preocupação em estruturar o curso a partir das exigências legais, mas sempre levando em consideração a realidade dos alunos pautou o caminho da Psicologia da Meto, denominação pela qual o curso ficou conhecido na comunidade interna e externa. Já em 1974 foi realizado um levantamento, coordenado pelo prof. Joel Camacho, para caracterizar o perfil dos alunos de Psicologia. O relatório resultante nos trouxe dados a respeito da história e desenvolvimento do curso que havia sido criado.

Também em 1975 foi criado o "Colegiado de Curso", como órgão integrador do curso, constituído pelo Coordenador de Curso, Chefes dos Departamentos, professores representantes dos Departamentos e representantes de alunos indicados pelo Centro Acadêmico de Psicologia.

A tradição de tomada de decisões colegiadas data do início do funcionamento do curso e desde sua criação são realizadas reuniões de seu corpo docente que, embora não tenham caráter decisório, sempre subsidiaram as decisões a serem tomadas pelo Colegiado.

A formação oferecida aos alunos ultrapassa os limites da Graduação. O Programa de Pós-Graduação em Psicologia iniciou suas atividades em 1978. No decorrer das décadas de 80 e 90, o Programa firmou-se na área de concentração da Psicologia da Saúde, tratando das questões comuns à Psicologia e à Saúde, definida como a aplicação dos conhecimentos e métodos da Psicologia na promoção e manutenção da saúde, na prevenção das doenças e no aperfeiçoamento do sistema de políticas públicas na área da Saúde. A integração de estudos e pesquisas entre graduação e pós-graduação tem-se mostrado um fator fundamental para a excelência dos profissionais formados pela Metodista.

Além da preocupação com a formação teórica de qualidade, o curso sempre buscou a formação prática de excelência. Para viabilizar o alcance deste objetivo, em 1975 foi criado o Setor de Psicologia 
Aplicada, responsável pela coordenação e realização dos estágios do Curso de Formação de Psicólogos, estrutura que funcionou até 1985.

A primeira responsável pelo Setor de Psicologia Aplicada, em 1975, foi profa Vera Lucia Wey, que permaneceu na função até o início de 1977. Seguiram a elaProf.JohannesPetrus Maria Berkers, de 1977 a 1981;Prof. Péricles de Oliveira Prado Filho, em 1982;ProfaDirce Beatriz Persson de Oliveira Prado, 1983 e, de 1984 a 1985,Profa. LucilaBenattiBertoline.

Nesse período, a direção da Faculdade de Ciências Humanas foi ocupada porClory Trindade de Oliveira (1971), Luiz Boaventura (1972 a 1974) e Ely Eser Barreto Cesar (1975). De 1983 a 1985,profa Hebe Ferreira Villela; de 1986 a 1989,Prof.Péricles de Oliveira Prado Filho; de 1990 a 1992 Prof.Dorival Rodrigues Beulke.

Em 1986 todos os cursos do Instituto Metodista de Ensino Superior passaram a funcionar com uma estrutura departamental e no curso foram estruturados dois departamentos: Departamento de Psicologia I e Departamento de Psicologia II. Deixa de existir o Setor de Psicologia Aplicada, e os estágios passaram para a coordenação dos Departamentos, designando-se três coordenadores de estágio: Profa. Marinês Santaroza Pereira, responsável pelo Estágio em Psicologia Clínica; Profa. Dirce Beatriz Persson de Oliveira Prado, para Psicologia Aplicada à Educação e Prof. Benedito Jorge Filho para Psicologia Aplicada à Indústria.

Entre 1993 e 1997, como parte da preparação para a instalação da Universidade, o curso de Psicologia passa a fazer parte da Faculdade de Ciências da Saúde, cujo diretor foi Prof. Antonio Alves Garcia, continuando como Coordenador o Prof. Péricles.

De 1997 a 1999 assume a coordenação do curso a prof. ․ Dra $^{-}$ Marília Martins Vizzotto. No final de 1998, com a transformação da Federação de Escolas Isoladas do ABC em Universidade Metodista de São Paulo, o curso de Psicologia passou a integrar a Faculdade de Ciências da Saúde e os departamentos de Psicologia I e II foram extintos. Em 1999 e até 2001, já na vigência da Universidade, Profa Marília assume a direção da Faculdade de Psicologia e Fonoaudiologia, acumulando a coordenação do curso de Psicologia.

Em 1997 foi publicado o primeiro número da revista PSICÓLOGO INFORMAÇÃO, que tinha como um de seus objetivos se 
tornar um veículo de divulgação de pesquisas científicas, atividades acadêmicas e reflexões críticas da Psicologia e áreas afins, contando com a contribuição de docentes e discentes da graduação do nosso curso. Seria um meio de estimular os estudantes ao gosto pela escrita científica. Além disso, a revista também dedicou-se à divulgação de trabalhos elaborados por docentes e discentes da pós-graduação e de profissionais de outras instituições.

Em 1999 foram constituídas, pela Coordenação do Curso, com anuência do seu Colegiado, cinco comissões compostas por professores do curso para estudo e avaliação das áreas e procedimentos pedagógicos, a saber: Comissão de Eventos; Comissão de Monitorias; Comissão de Avaliação Interna; Comissão de Estudo do Setor de Psicologia Aplicada e Comissão de Estudos Curriculares. Estas comissões propuseram medidas compatíveis com uma concepção mais atualizada sobre o ensino da Psicologia e desempenharam um papel importante na transformação de vários aspectos tanto da estrutura quanto da dinâmica do Curso.

Em 1999, o Curso de Psicologia passou por grande transformação, tendo em vista as novas Diretrizes Curriculares para os cursos de Psicologia no Brasil definidas por uma Comissão de especialista do MEC. Na mesma época, o curso passou a integrar a Faculdade de Psicologia e Fonoaudiologia.

Nessa mesma época a direção do curso de Psicologia buscou assessoria externa para adaptação do curso às novas diretrizes e a convidada foi Profa. Dra Carolina M. Bori, escolha justificada pelo fato da referida professora ter sido membro da Comissão de Especialistas, designada pelo MEC para a tarefa de formulação das Diretrizes Curriculares para os cursos de Psicologia.

Uma comissão de professores do curso participou do debate nacional acerca das Diretrizes Curriculares com a Comissão de Especialistas, bem como dos encontros promovidos pela Associação Brasileira de Ensino de Psicologia (ABEP), ocorridos em Salvador e Brasília, a fim de integrarem-se no debate sobre as Diretrizes Curriculares Nacionais, sendo discutidos os seguintes indicadores: núcleo comum, habilidades e competências, ênfases, eixos estruturantes, princípios e compromissos, estágios (básico e específico), entre outros. 
Cabe ressaltar que a coordenação de curso, o corpo docente e discente participaram ativamente de debates e simpósios culminando, inclusive, na realização de um evento, a XXIII Semana de Psicologia, dedicada exclusivamente à discussão deste tema.Para maior divulgação das medidas que seriam adotadas, foi publicado um artigo denominado: A definição de diretrizes curriculares em uma estruturação curricular em Psicologia e o estágio de formação profissional, de autoria da Prof ${ }^{a}$.Dra. Maria Amélia Matos, na revista Psicólogo in Formação, ano IV, no 4, Jan. - Dez. 2000, p. 11 - 24 .

Em 2000, o Colegiado do Curso aprovou a transformação do Setor de Psicologia Aplicada (SAP), em Núcleo de Estudos, Pesquisas e Atendimento em Psicologia (NEPAP), buscando integrar estágios e atividades práticas ao longo dos cinco anos do curso, bem como promover, de modo mais efetivo, o intercâmbio de estudos e pesquisas com a Pós-Graduação (Strictu e Lato Sensu), sempre com a finalidade de fundamentar e ampliar a formação em Psicologia.

Ao final de 2001 a prof $^{\underline{a}}$ Elaine de Oliveira assume a direção da Faculdade e o curso de Psicologia instala-se no Campus Planalto; a professora permaneceu até 2005.

Nova proposta curricular para o curso é apresentada em 2004, contemplando as novas Diretrizes Pedagógicas da Universidade Metodista de São Paulo para todos os seus cursos aprovadas pelo Conselho Universitário (CONSUN).

Em 2006, prof ${ }^{a}$ Dra Hilda Rosa Capelão Avoglia (2006-2008) assume a direção da Faculdade de Psicologia e Fonoaudiologia.

Durante muitos anos vigorou, na estrutura curricular dos cursos da Metodista, a concepção de disciplinas como unidades de ensino. Contudo, a resolução CONSUN 39/2007 requereu a revisão do termo "disciplina", que se relacionava apenas ao contexto da produção e da reprodução do conhecimento, passando a vigorar a terminologia e conceito de Módulos, que ampliava a relação ensino aprendizagem. Uma nova proposta do Projeto Pedagógico Curso (PPC) foi apresentada, em 2008, pelo Colegiado do curso de Psicologia,tendo como principal mudança os conteúdos curriculares organizados em módulos. Nesta nova proposta, foram mantidos tanto as ênfases (Psicologia Comunitária e da Saúde e Psicologia Organizacional e do Trabalho) quanto os estágios básicos e específicos. 
A partir de 2009, ocorreu uma mudança na estrutura de faculdades na Universidade Metodista de São Paulo. A Faculdade de Psicologia e Fonoaudiologia deixou de existir e tanto o curso de graduação em Psicologia quanto o curso de Pós-Graduação em Psicologia da Saúde passaram a integrar, juntamente com outros cursos, a Faculdade da Saúde. O Prof. Dr. Rogério Gentil Bellot assume, então, a direção da Faculdade.

Em 2010 os estágios específicos da formação de psicólogo - estágios de Psicologia Aplicada a Clínica (Triagem, Psicodiagnóstico e Psicoterapia) passaram a acontecer na Policlínica (Campus Rudge Ramos), dentro de uma nova concepção de atendimento integrado, que reunia os serviços de Psicologia, Nutrição, Fonoaudiologia, Fisioterapia, Odontologia e Educação Física.

A partir de 2012 o Colegiado de curso de Psicologia e a direção da Faculdade consideram a necessidade de realizarem uma série de discussões sobre aspectos contemporâneos e demandas atuais a serem inseridas no Projeto Pedagógico do Curso.

Em 2013, o Núcleo Docente Estruturante (NDE) e a direção da Faculdade realizaram uma série de reuniões e discussões para a implantação do projeto de Formação de Professores de Psicologia, complementar ao Projeto Pedagógico do Curso de Formação de Psicólogo, sempre em consonância com as Diretrizes do MEC..

O Colegiado de Curso de Psicologia apresentou em 2014 uma nova proposta do Projeto Pedagógico Curso (PPC). A proposta de reformulação considerou os pilares anteriores da concepção de formação do psicólogo por meio das ênfases em: (1) Psicologia Organizacional e do Trabalho e (2) Psicologia da Saúde e Comunitária.

Em 2014 o CONSUN aprova a nova estrutura da Universidade Metodista de São Paulo e o curso de Psicologia integra a Escola de Ciências Médicas e da Saúde, ainda sob a direção do prof. Dr. Rogério Gentil Bellot.

Este breve retrospecto histórico mostra que o Curso de Psicologia da Universidade Metodista busca, desde sua implantação, o estabelecimento de uma dinâmica que garanta a democratização das decisões e que contribua para o desenvolvimento de uma cultura de abertura e amplitude, que assegure as mudanças essenciais para a formação de profissionais comprometidos com sua realidade social. 
Além da formação científica e profissional, o curso de Psicologia da Universidade Metodista assume como diretriz a promoção humana e o compromisso com a comunidade na qual a instituição se insere. Nesta trajetória, destacam-se a parceria com a Pós-Graduação; parceria / promoção do curso em inúmeros eventos nacionais e internacionais de natureza científica; participação em ações comunitárias de âmbito regional e nacional, sempre com a presença de docentes, discentes e ex-alunos. Além disso, sempre houve participação dos professores, tanto interna como externamente, em congressos e encontros com outras instituições educacionais e profissionais e os Conselhos - Regional e Federal de Psicologia, discutindo, e trazendo contribuições para o curso e na realização das Semanas Culturais de Psicologia sempre houve a preocupação de trazer profissionais que muito contribuem para a realidade acadêmica.

Em seu percurso de 45 anos, o curso de Psicologia sempre preservou sua memória sem, contudo, desconsiderar o diálogo com a realidade social/regional / nacional, pois compreende que este é o caminho para o planejamento e a construção de sua perspectiva futura respeitando a tradição e sempre em busca da inovação.

Contato da autora:

Mariantonia.chippari@metodista.br

Recebido em : 12/04/2016

Aceito em: 05/05/2016 